\title{
Régulation fluviale par commande prédictive avec modèle de connaissance embarqué
}

\section{River regulation through predictive control with all on-board knowledge model}

\author{
par J.C. Pagès, J.M. Compas, P. Roumieu, D. Roult \\ Compagnie Nationale du Rhône
}

J. Sau

Institut des Sciences et Techniques de l'ingénieur de Lyon

All hydraulic structure designs now make use of mathematical models.

Knowledge based models use a discrete form of non-linear Barré-de-St-Venant equations describing onedimensional free surface flows. Based on a structure of complex topological data, once calibrated, they permit simulating the hydraulic behaviour of rivers and calculating the levels and flows at each point.

The capacities of current computers now make it possible to use a knowledge-based model of hydraulic structures. Such a model embedded in a structure control computer permits implementing a robust predictive command capable of taking into account estimated future changes as trends, thus increasing control performance.

Different types of embedded model management can be used to formulate the control (scenario management, identification of an adaptative linear model, etc.).

The needs of overall management of volumes during floods are now leading to consideration on the possibilities of centralising the operation of a chain of developments. The feasibility of such a system has been studied for the dams of the Rhone downstream of Lyon.

\section{I $\square$ INTRODUCTION}

Depuis maintenant de nombreuses années, des méthodes numériques ont été mises au point pour intégrer les équations non-linéaires de l'hydraulique à surface libre. Sur ces bases, des programmes ont été développés, permettant de modéliser les écoulements en rivières et canaux, dans des conditions chaque fois plus complexes. Notamment, l'introduction de lois hydrauliques spécifiques aux divers ouvrages et organes de contrôle ont permis de généraliser l'utilisation de modèles mathématiques pour les études des écoulements à surface libre dans les cours d'eau.

Pour l'exploitation, avoir un modèle «à jour " de l'aménagement permet, entre autres, de l'utiliser comme modèle interne dans une boucle de régulation du type commande prédictive ou commande optimale. La commande prédictive de type PFC, développée par Richalet |1| de l'Adersa, permet dans un premier temps d'intégrer facilement un modèle mathématique en lieu et place de l'habituel modèle linéaire simplifié (gestion de scénarios). Par la suite, il est possible d'intercaler entre le code de simulation des écoulements et la commande prédictive PFC un modèle linéaire adaptatif identifié systématiquement généré. Cela élargit le champ des possibilités d'un tel système de régulation en permettant par exemple de réaliser un système de conduite coordonnée prédictive d'une chaîne d'aménagements.

Dans les chapitres qui suivent, nous présenterons d'abord le logiciel de simulation des écoulements à surface libre développé à la CNR. Ensuite, I'utilisation de ce code comme modèle interne d'un régulateur par commande prédictive sera décrite. Enfin, la perspective d'utiliser une commande prédictive multi-variable pour une conduite coordonnée d'une chaîne d'aménagements sera envisagée.

\section{II —CRUE 7 : LOGICIEL DE SIMULATION DES ÉCOULEMENTS À SURFACE LIBRE}

L'essor des performances de l'informatique et la généralisation de l'analyse numérique ont permis ces vingt dernières 
années une plus large diffusion des codes de simulation du comportement des fluides.

Dans le domaine de l'hydraulique à surface libre, de nombreux produits existent. Ils sont pour la plupart basés sur une intégration numérique des équations de Barré de SaintVenant appliquée le long de biefs séparés par des profils en travers permettant la meilleure représentation physique du tronçon à modéliser.

Ces équations sont une restriction monodimensionnelle des équations de Navier-Stokes décrivant des écoulements fluides quelconques. Elles sont au nombre de deux. Leur forme différentielle, prise en compte pour la discrétisation et la programmation des modules de calcul est la suivante : continuité :

$$
L \cdot \frac{\partial Z}{\partial t}+\frac{\partial Q}{\partial x}=q
$$

dynamique :

$$
\frac{\partial Q}{\partial t}+\frac{\partial\left(\beta \cdot \frac{Q^{2}}{S}\right)}{\partial x}+g \cdot S \cdot \frac{\partial Z}{\partial x}+g \cdot S \cdot J_{e}=\left(u_{q} \pm \frac{Q}{S}\right) \cdot q
$$

où $Q$ représente le débit de la rivière $\left(\mathrm{m}^{3} / \mathrm{s}\right), Z$ la cote du plan d'eau $(\mathrm{m}), t$ le temps (s), $S$ la surface mouillée perpendiculairement à l'écoulement $\left(\mathrm{m}^{2}\right), L$ la largeur au plan d'eau de la section $(\mathrm{m}), J_{e}$ la pente de la ligne d'énergie, $q$ le débit de ruissellement le long des biefs $\left(\mathrm{m}^{2} / \mathrm{s}\right), u_{q}$ la vitesse du débit de ruissellement $(\mathrm{m} / \mathrm{s}), \beta$ le coefficient de distribution de la vitesse.

L'expression communément admise pour le calcul de la pente de la ligne d'énergie est celle de Manning-Strickler :

$$
J_{e}=\frac{Q^{2}}{S^{2} \cdot K^{2} \cdot R_{h}^{4 / 3}}
$$

où $K$ représente le coefficient de Strickler (égal à l'inverse du coefficient de Manning), et $R_{h}$ le rayon hydraulique de la section perpendiculaire à l'écoulement $(\mathrm{m})$.

Notons que le coefficient de Strickler $(K)$ intègre non seulement la rugosité du fond mais aussi toutes les pertes de charge réparties le long de la rivière ou du canal qu'il est difficile de localiser et de modéliser. Ce coefficient doit plutôt être considéré comme un coefficient de calage du modèle.

Le logiciel CRUE entièrement développé à la Compagnie Nationale du Rhône, simule le comportement hydraulique d'une rivière ou d'un système de canaux. Le code de calcul permet de représenter des réseaux ramifiés ou maillés en régime permanent ou transitoire. Il utilise la méthode des différences finies avec un schéma d'intégration semi-implicite inconditionnellement stable dit de Preismann (voir Cunge [2]). L'introduction de zones de stockage (appelés casiers) reliées entre elles par des lois hydrauliques variées permet de simuler des situations particulièrement complexes.

Deux types de données en entrée peuvent être différenciés : - Les données topographiques et de calage :

CRUE s'appuie sur le concept de BRANCHES et celui de NEUUS :

- une branche représente une zone de l'écoulement régie par un seul type de loi hydraulique, comme :

- branche fluviale constituée de profils en travers ;

- seuil ;

- orifice et clapet ;

- vannes réglables ;

- vannes de régulation automatiques (niveau amont ou aval

constant, niveaux associés,...);

- perte de charge - loi $D z=f(Q)$;

- barrage effaçable ;

- loi type station de pompage ;

- loi Strickler.

- Un nœud désigne la jonction entre plusieurs branches.

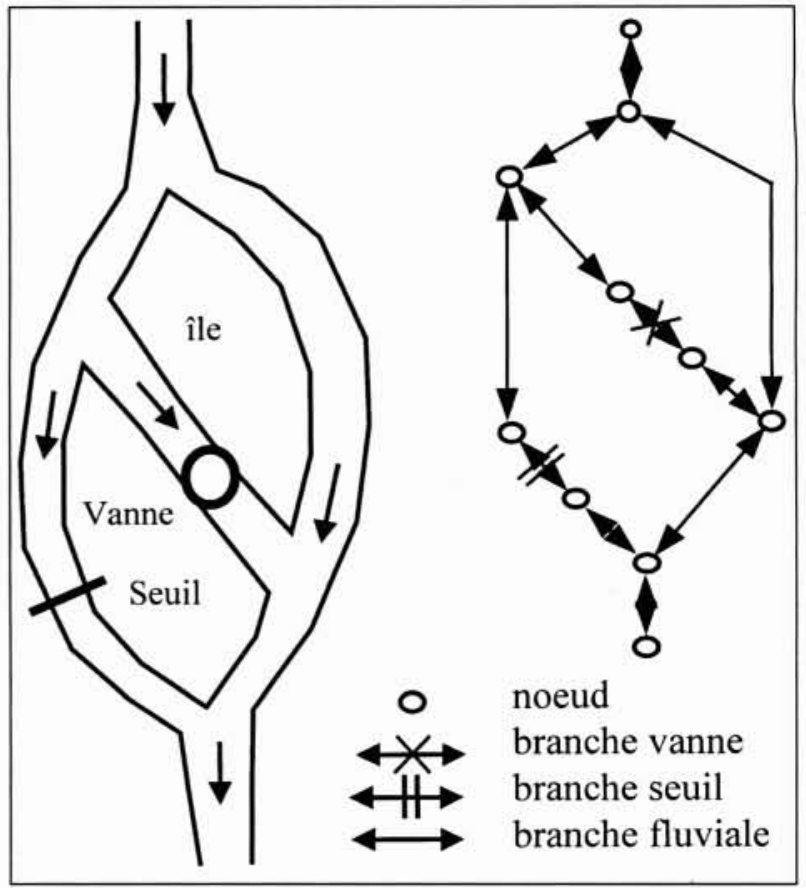

1. Exemple de modélisation.

L'écoulement dans une branche fluviale est conditionné par un nombre quelconque de profils en travers.

Les coefficients de rugosité sont introduits sous forme de coefficients de Strickler définissables pour chaque profil et variables en fonction de la profondeur d'eau.

Les limites de lits et de champs d'inondation sur chaque profil, ainsi que des casiers peuvent être également introduits pour modéliser au mieux le comportement d'une rivière.

- Les données de simulations hydrauliques:

Une ligne d'eau, supposée ou imposée, est à fournir en tant qu'état initial. Les conditions aux limites, à introduire aux extrémités du modèle, peuvent être, soit des limnigrammes $Z(t)$ ou des hydrogrammes $Q(t)$ fonction du temps, soit des lois $Q(Z)$ ou $Z(Q)$, soit des conditions d'asservissement pour simuler une régulation par exemple.

Le programme comprend deux parties, une partie lecture et vérification de la cohérence des données et la partie calcul proprement dite qui permet de générer des états permanents successifs et des états transitoires.

Le pas de temps peut être variable au cours d'une simulation. La méthode numérique est suffisamment robuste pour pouvoir prendre en compte des phénomènes « lents » (type crue de durée de un ou plusieurs jours) ou au contraire très rapides (propagation d'ondes liées à des phénomènes de disjonction).

Les données en sortie sont extraites de fichiers résultats générés par l'application. Une batterie de modules accèdent à ces fichiers et présentent les données sous forme de tableaux ou de tracés. Ces derniers utilisent une bibliothèque graphique multi-périphériques permettant un portage aisé et compatible avec la plupart des matériels existants.

Les données de sorties peuvent être :

- les niveaux et débits au droit de chaque profil mais aussi la section mouillée, la vitesse moyenne, $V^{2} / 2 \mathrm{~g}$, la charge, la vitesse critique, la force tractrice,...) ;

- les profils en travers avec le plan d'eau ;

- les lois $Q(H)$;

- le profil en long des lignes d'eau ;

- les hydrogrammes et limnigrammes ;

- les schémas topologiques :

- les caractéristiques hydrauliques des sections.

Les graphes suivants fournissent quelques exemples de sorties du logiciel CRUE. 


\section{에}
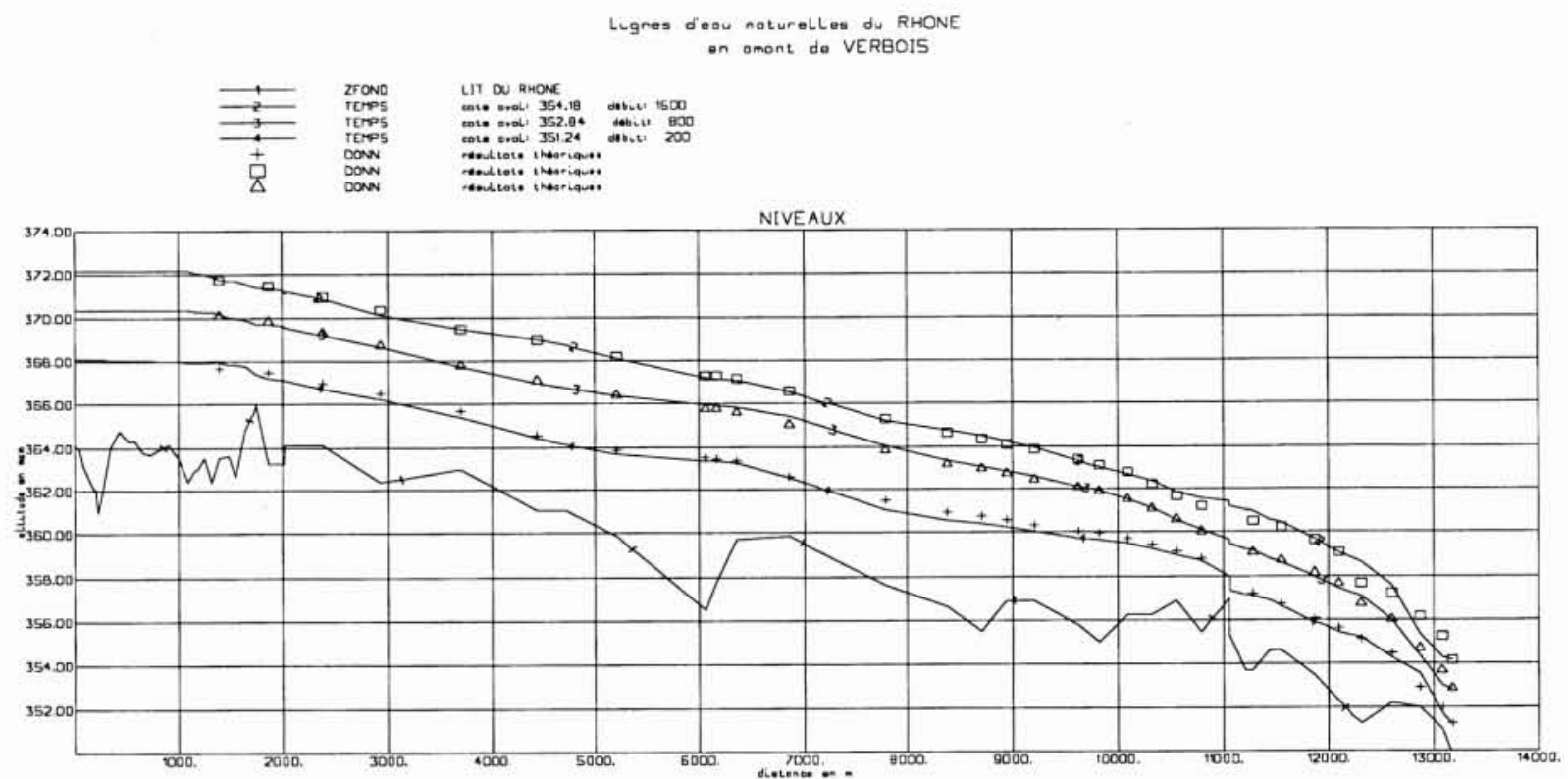

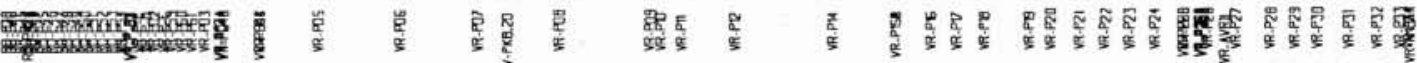

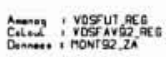

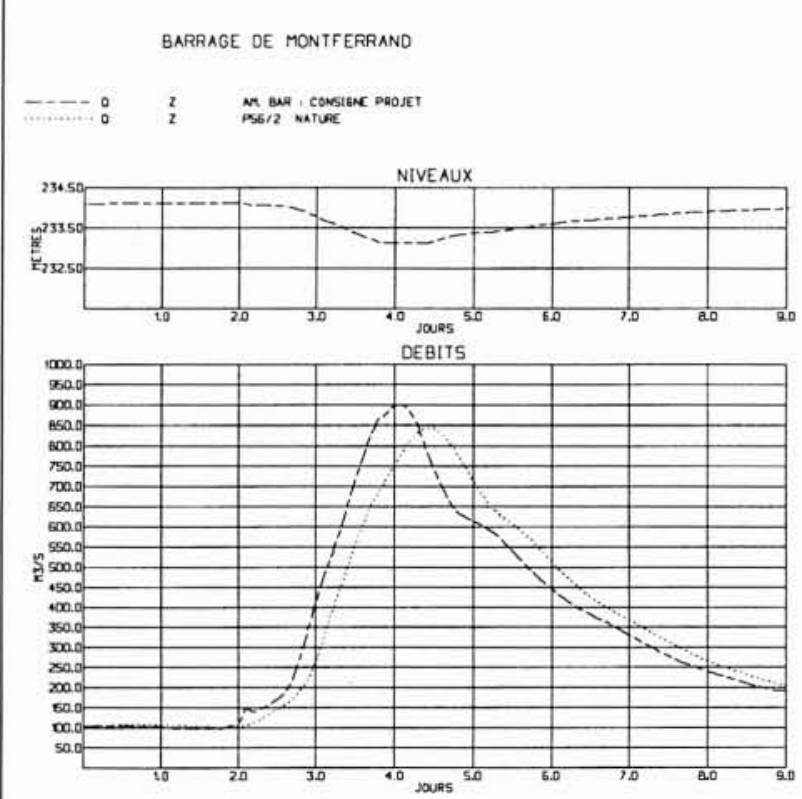

BIEF DE CHOISEY

LOI $O / H$ AVANT ET APRES AMENAGEMENT

$\begin{array}{ll} & \text { Etat naturel } \\ -\ldots- & \text { Etat amenage }\end{array}$

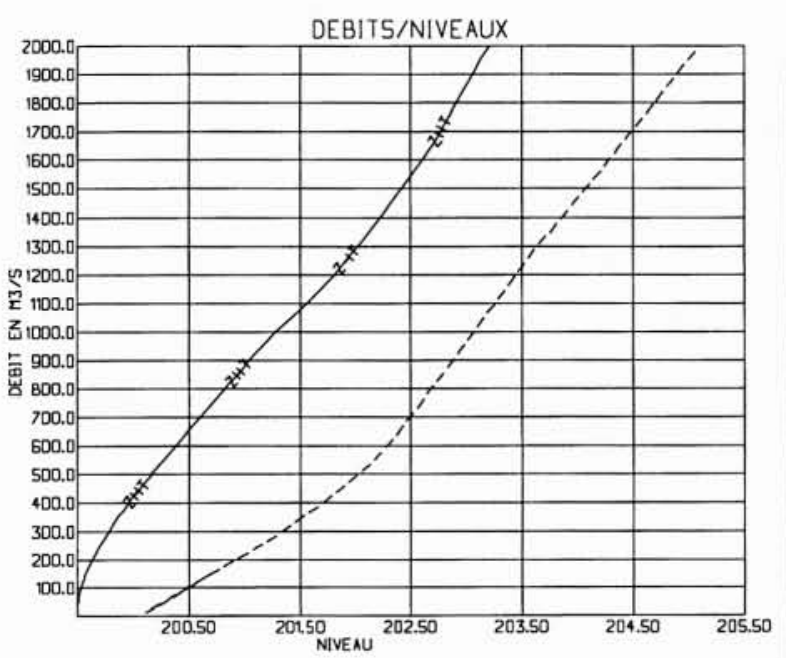

2. Exemples des sorties graphiques du logiciel CRUE.

La bibliothèque de modules de simulations hydrauliques et le logiciel CRUE ont été conçus de manière très modulaire de façon à pouvoir répondre à différents besoins des utilisateurs (voir Roult [3]). Développés et maintenus en interne, ils peuvent être constamment enrichis en fonction des demandes de l'exploitation du Rhône ou des clients de la Direction Ingénierie de la CNR.

Ils forment un ensemble complet d'outils pour mener à bien toutes les étapes nécessaires à la conception et au dimensionnement d'un aménagement hydraulique, ou a pos- 
teriori, pour réaliser une étude hydraulique au voisinage d'ouvrages existants.

De plus, l'actualisation en permanence d'un modèle mathématique permet de développer des outils de simulations et d'aide à la décision. Ainsi les exploitants peuvent apprécier quel sera le résultat d'une action donnée sur le comportement du système dont ils sont gestionnaires.

Par exemple, le contrôle de cohérence des programmes de débits des centrales hydroélectriques du Rhône est réalisé grâce à CRUE.

Un second exemple, qui fera l'objet des chapitres suivants, est l'utilisation du logiciel comme modèle embarqué dans le calculateur de régulation des aménagements d'un cours d'eau. Le code servira alors de modèle de connaissance pour un régulateur issu de l'automatique avancée (voir Pagès [4] et Landau [5]).

\section{DÉGULATION PAR COMMANDE PRÉDICTIVE AVEC MODÈLE MATHÉMATIQUE EMBARQUÉ}

\subsection{Principes de régulation d'une retenue}

L'aménagement du Rhône est à buts multiples (production électrique, navigation, gestion de l'eau, irrigation et loisirs). Le fleuve a été pour cela divisé en paliers conçus pour la plupart suivant une architecture type. Une dérivation du lit du fleuve comprend une centrale de production électrique ainsi qu'une écluse grand gabarit. La retenue est créée par un barrage évacuateur de crues sur le bras de fleuve courtcircuité juste à l'amont de la dérivation.

La régulation est réalisée par le réglage d'un débit sortant : celui de l'usine hydroélectrique si le débit est inférieur au débit maximum admissible de la centrale (conduite en programme de débit en période énergétique) ou celui du barrage si le débit est supérieur au débit maximum admissible de la centrale (régulation de niveau en période de crue).

\subsection{Présentation de la commande prédictive}

L'algorithme traditionnellement utilisé en régulation fluviale est un classique correcteur PI (Proportionnel, Intégral) ou PID avec une action Dérivée ou bien une anticipation sur les perturbations mesurées. Les phénomènes non-linéaires avec retards purs variables du processus considéré impliquent un réglage optimal difficile des coefficients du régulateur PID. Le recalage de ces derniers suivant l'état de la retenue est une solution pour obtenir un jeu de réglage «fin " de la régulation. Cette solution a été appliquée pour le Rhône Suisse dans le cadre d'une prestation d'ingénierie de la CNR (voir Lagoutte [6]).

Pour le Rhône en aval de Lyon, la CNR a choisi d'implémenter en période de crue, une régulation par commande prédictive en embarquant le modèle mathématique CRUE dans le calculateur d'aménagement.

La stratégie de commande prédictive de type PFC - Predictive Functional Control - développée par Richalet [1] de l'Adersa est ici présentée et appliquée. Son principe réside sur l'usage du modèle interne en déterminant, à chaque pas de calcul $-n-$, la commande optimale permettant le respect d'une trajectoire de référence dans une fenêtre glissante du futur appelée horizon de coïncidence (fig. 5).

L'élaboration de la commande de base est faite à chaque pas de régulation suivant les étapes décrites ci-après (voir Compas [7]) :

- Recalage du modèle embarqué et détermination des estimateurs de biais.

Le modèle de connaissance basé sur une approximation des lois physiques du phénomène simule bien le processus. Cependant, un modèle complètement indépendant de la réalité présenterait un biais croissant étant donné qu'il existe des erreurs de structure (provoquées par exemple par la discrétisation spatiale) et des erreurs d'état (dues à l'imperfection des mesures et aux perturbations inconnues).

Le recalage consiste à imposer au modèle mathématique des conditions aux limites surabondantes, des niveaux mesurés en l'occurrence. Elles génèrent automatiquement, au droit de leurs points d'application, des débits d'injection (positifs ou négatifs) dits de correction qui constituent autant d'estimateurs non-linéaires de biais images des diverses erreurs existantes entre modèle et réalité.

Ces débits de correction sont ensuite pris en tendance dans la prédiction. Leur suivi permet en outre de contrôler en temps réel l'évolution des erreurs et donc l'état de dégradation du système global (ensemble modèle/régulateur). Ils constituent ainsi des indicateurs précieux dans le cadre d'une maintenance préventive pour l'exploitant.

- Détermination des réglages du régulateur.

- Horizon de prédiction.

Le principe de commande est basé sur une exploration du futur rendue possible par la mise à disposition du modèle. La durée de cette exploration temporelle est appelée horizon de prédiction $-\mathrm{H}-$.

- Trajectoire de référence.

Une trajectoire de référence $y_{\text {ref }}$ est définie dans le futur pour rallier la consigne. Un raccordement de type exponentiel donne satisfaction. La constante de cette exponentielle - coefficient $\lambda-$ règle le temps de réponse en boucle fer-

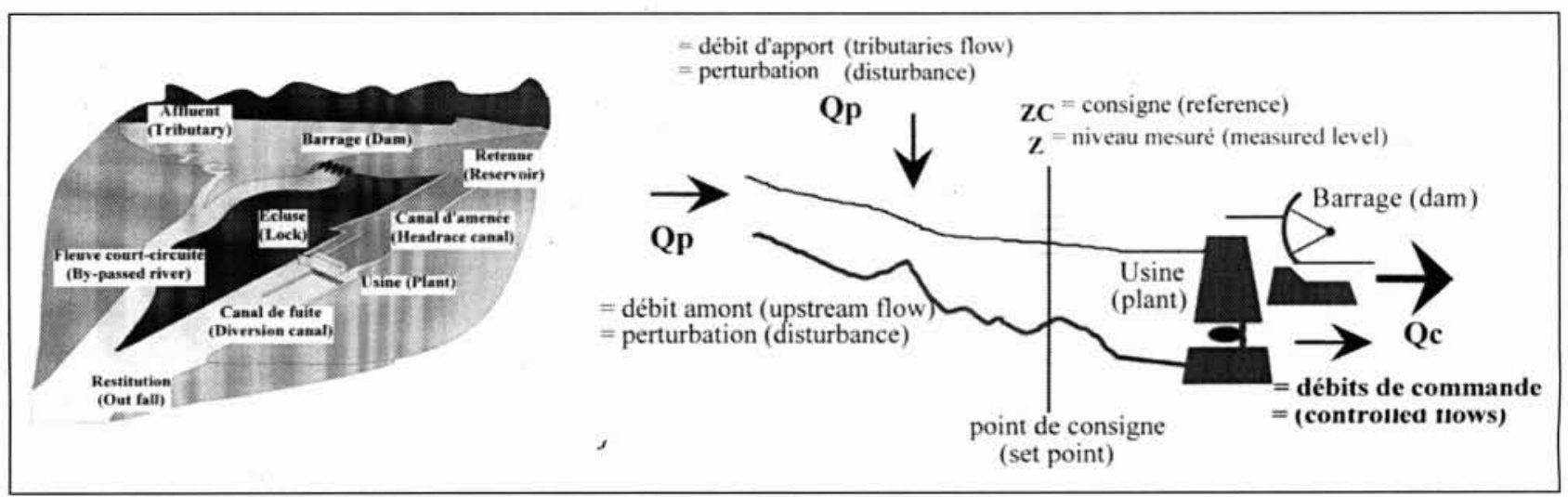

3. Présentation de la régulation d'un aménagement du Rhône. 


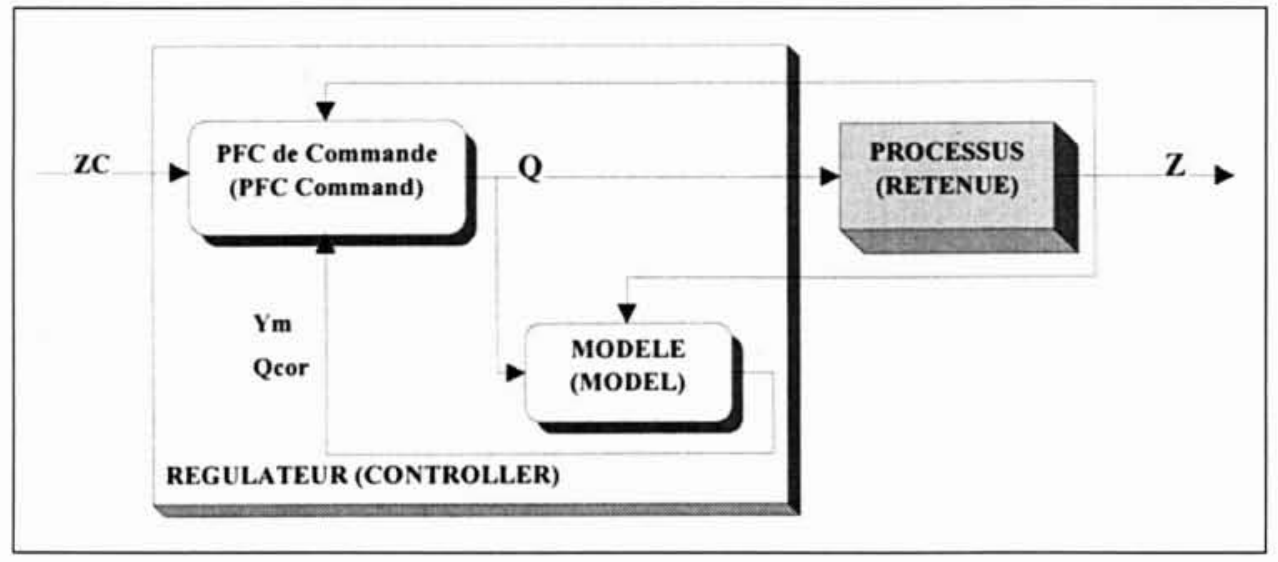

4. Schéma de commande prédictive à modèle interne recalé.

mée et donc la dynamique. Elle s'exprime (avec $n$ l'instant présent et $j=0$ à $H$ ) :

$$
y_{r e}(n+j)=Z C(n+j)-\lambda \cdot(Z C(n)-Z(n))
$$

- Horizon de coïncidence et points de coïncidence.

Sur cette trajectoire, des points particuliers dits de coïncidence sont choisis à des temps $h_{\mathrm{s}}$ futurs et servent de cibles pour la trajectoire à calculer par le modèle. Ces cibles sont approchées par une méthode de minimisation de critère de distance, les moindres carrés par exemple. Bien entendu, leur nombre influe sur la précision du régulateur mais est directement lié à la structuration des lois de commande comme nous le verrons plus loin.

La plage temporelle où sont situés tous les points de coïncidence est appelée horizon de coïncidence $-\mathrm{HC}-$.

Bien entendu $H C$ doit être compris dans $H$ et doit être tel que la commande soit représentative de la dynamique du processus, en particulier être au-delà du temps de retard pur. Son choix influe surtout sur la robustesse et à un degré moindre sur la dynamique de la réponse.

- Structuration de la commande future.

Dans un objectif de simplification de l'optimisation et pour garantir l'unicité de la solution, il est important de structurer la loi de commande. Ainsi avec une période d'échantillonnage Te et des fonctions de base unitaires définies comme suit,

degré 0 : échelon unitaire $k=0 \Rightarrow U B_{0}(j)=1$,

degré 1 : rampe unitaire $k=1 \Rightarrow U B_{1}(j)=j . T e$,

degré $N$ :

$k=N \Rightarrow U B_{N}(j)=(j . T e)^{N}$.

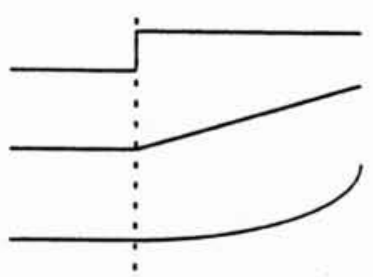

la loi de commande sur l'horizon de prédiction peut être structurée de façon polynomiale :

$$
\Delta Q c(n+j)=\sum_{s=0}^{N} \mu_{s}(n) \cdot U b_{s}(j)=\mu(n)^{T} \cdot U b(j)
$$

avec $n$ l'intant présent, $j=0$ à $H$,

et avec

$$
\begin{aligned}
& \mu(n)^{T}=\left(\begin{array}{llll}
\mu_{0}(n) & \mu_{1}(n) & \ldots & \mu_{M}(n)
\end{array}\right) \text { et } \\
& U b(j)^{T}=\left(\begin{array}{lllll}
U b_{0}(j) & U b_{1}(j) & \ldots & U b_{M}(j)^{\prime}
\end{array}\right.
\end{aligned}
$$

$N$ est le degré du polynôme et $\mu(n)$ le vecteur de $N+1$ inconnues définissant la commande recherchée. Le nombre de points de coïncidence doit être au moins égal à $N+1$ pour permettre la résolution. Le choix de $N$ permet d'optimiser la précision.

Les paramètres de réglage à déterminer sont donc au nombre de trois et ont une action relativement découplée sur les caractéristiques de la régulation. Tout d'abord, il faut choisir le nombre de fonctions de base $N+1$ en général égal au nombre de points de coïncidence. Il influe uniquement sur la précision du régulateur. Ensuite, il faut choisir le coefficient $\lambda$ de la trajectoire de référence agissant sur la dynamique et dans une moindre mesure la robustesse. Finalement, il reste à déterminer la position des points de coïncidence influant sur la robustesse et un peu la dynamique.

Les autres valeurs présentées plus haut se déduisent de ce choix de trois paramètres ayant une représentation physique précise.

\section{- Calcul de la commande.}

L'objectif est de déterminer la meilleure commande permettant de rallier la trajectoire de référence en visant les points de coïncidence dans l'horizon $\mathrm{HC}$. La figure 5 schématise cette stratégie. La commande peut être obtenue par deux voies possibles que nous décrivons plus bas.

- Calcul de la commande par gestion de scénarios.

Le modèle de connaissance embarqué n'est pas linéaire et ne permet donc pas l'obtention d'une loi de commande analytiquement.

La difficulté peut être contournée, pour les applications à commandes monovariables ou successives comme avec les ouvrages du Rhône, par une stratégie de prédiction basée sur la gestion itérative de scénarios de commande conduisant à une convergence progressive.

Dans ce cas un seul point de coïncidence est choisi à un temps $n+h_{0}$ avec $h_{0}=H$. La structuration de la commande est limitée au degré $N=0$, soit l'échelon de base unitaire :

$$
U b_{0}(j)=1 \Rightarrow \Delta Q c(n+j)=\mu(n)
$$

avec $j=0$ à $H=h_{0}$ (incrément de commande).

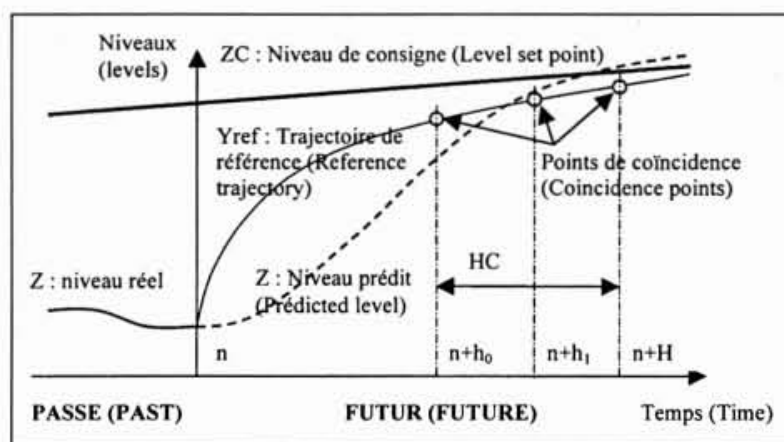

5. Stratégie de commande prédictive. 
Des simulations en boucle ouverte sont menées sur le modèle embarqué recalé en appliquant des scénarios de loi de commande de débit. Les sorties en niveau à l'horizon de prédiction $H=h_{0}$ sont enregistrées :

- scénario 1: loi de commande appliquée : $Q c_{1}(n+j)=0$, $j=0$ à $h_{0} \Rightarrow$ sortie lâchée : niveau $Z_{1}\left(n+h_{0}\right)$;

- scénario 2: loi de commande appliquée : $Q c_{2}(n+j)=$ cste, $j=0$ à $h_{0} \Rightarrow$ sortie forcée : niveau $Z_{2}\left(n+h_{0}\right)$.

Avec l'hypothèse de linéarité au $1^{\mathrm{er}}$ ordre près autour d'un régime de fonctionnement, la loi de débit est calculée par la relation :

$$
\begin{gathered}
\mu(n)=\Delta Q c(n)=\frac{y_{r e}\left(n+h_{0}\right) \pm Z_{1}\left(n+h_{0}\right)}{Z_{2}\left(n+h_{0}\right) \pm Z_{1}\left(n+h_{0}\right)} \cdot\left(\Delta Q c_{2}(n) \pm \Delta Q c_{1}(n)\right) \\
\Delta Q_{c}(n)=Q_{c}(n) \pm Q_{c}(n \pm 1) \\
\Delta Q_{c 1}(n)=Q_{c 1}(n) \pm Q_{c 1}(n \pm 1) \text { et } \\
\Delta Q_{c 2}(n)=Q_{c 2}(n) \pm Q_{c 2}(n \pm 1)
\end{gathered}
$$

Cette loi permet d'approcher le point de coïncidence $y_{r e f}(n+$ $h_{0}$ ) situé sur la trajectoire de référence. Par itérations et scénarios successifs et en s'appuyant sur la méthode de la sécante, la loi de commande recherchée est ainsi obtenue.

C'est cette commande qui est appliquée au processus pour le pas en cours. Le cas échéant, une prise en compte de contraintes sur la commande ou sur la sortie est aisée.

- Calcul de la commande par l'intermédiaire d'un modèle linéaire adaptatif associé.

La stratégie précédente pénalise la souplesse de la commande prédictive, en particulier dans une approche multivariables et pour des réglages plus complexes (prise en compte de plusieurs fonctions de base). Dans un tel cas, l'usage temps réel d'un modèle linéaire adaptatif, lié au modèle non-linéaire embarqué, évite cette limitation et nous ouvre alors l'accès à toutes les ressources et potentialités de la commande prédictive.

A chaque pas de temps et autour d'un régime de fonctionnement courant, un modèle linéaire est extrait du modèle non-linéaire précédemment recalé. Il s'appuie sur les résultats de scénarios (un libre et un forcé par entrée) appliqués à ce dernier et s'écrit avec une représentation de type ARMA:

$$
\Delta Z(n)=\Delta Z(n \pm 1)+\sum_{s=1}^{T} p_{s} \cdot \Delta Q p(n \pm s)+\sum_{s=1}^{T} c_{s} \cdot \Delta Q c(n \pm s)
$$

avec $\Delta Z(n)$ incrément de niveau entre les instants $n$ et $n-1$, $\Delta Q o(n-s)$ et $\Delta Q c(n-s):$ respectivement, incréments de débit de commande et de perturbation entre les instants $n-s$ et $n-s-1$.

Une simple transformation nous donne alors un modèle sous une pseudo forme d'état de dimension $T$ :

$$
\left\{\begin{array}{l}
X(n)=A \cdot X(n \pm 1)+B p \cdot \Delta Q p(n \pm 1)+B c \cdot \Delta Q c(n \pm 1) \\
\Delta Z(n)=C \cdot X(n)
\end{array}\right.
$$

ce qui nous donne pour l'instant futur $n+j$

$$
\begin{gathered}
\Delta \mathrm{Z}(n+j)=\underbrace{\Delta Z_{\text {force }}(n+j)}_{\begin{array}{c}
\Delta Z_{\text {lachê }}(n+j) \\
+C \cdot A^{j} \cdot X(n)+C \cdot \sum_{s=1}^{j} A^{s \pm 1} \cdot B c \cdot \Delta Q c(n+j \pm s)
\end{array}}
\end{gathered}
$$

La partie lâchée de $\Delta Z$ est obtenue directement par le modèle de connaissance avec le scénario dit également lâché $\left(\Delta Q c_{1}=0\right)$.

Pour une entrée de base $U b_{k}(j)$, la partie forcée vaut :

$$
Y b_{k}(j)=C \cdot \sum_{s=1}^{j} A^{s \pm 1} \cdot B c \cdot U b_{k}(j \pm s)
$$

Le principe de superposition permet d'en déduire la sortie globale forcée $\Delta Z_{\text {forcé }}$ correspondant à la structure polynomiale de base choisie en entrée :

$$
\begin{gathered}
\Delta Q c(n+j)=\sum_{s=0}^{N} \mu_{s}(n) \cdot U b_{s}(j)=\mu^{T}(n) \cdot U b(j) \\
\Rightarrow \Delta Z_{\text {forcé }}(n+j)=\sum_{s=0}^{N} \mu_{s}(n) \cdot Y b_{s}(j)=\mu^{T}(n) \cdot Y b(j)
\end{gathered}
$$

L'objectif est de déterminer les commandes futures $\Delta Q c(n+j)$ sur $H$ tel que le critère ci-dessous soit minimal :

$$
\begin{aligned}
J(n)=\sum_{s=0}^{N}\left(\Delta Z\left(n+h_{s}\right) \pm y_{\text {ree }}\left(n+h_{s}\right)\right)^{2} & =\sum_{s=0}^{N}\left(\Delta Z_{\text {laché }}\left(n+h_{s}\right)\right. \\
& \left.+\Delta Z_{\text {force }}\left(n+h_{s}\right) \pm y_{\text {red }}\left(n+h_{s}\right)\right)^{2}
\end{aligned}
$$

En injectant (1) dans ce critère, il est facile de calculer la valeur $\tilde{\mu}(n)$ solution de

$$
\frac{\partial J(n)}{\partial \mu(n)}=0 .
$$

La commande recherchée pour l'instant présent $-n-$ est égale à :

$$
\Delta Q c(n)=\tilde{\mu}^{T}(n) \cdot U b(0)=\tilde{\mu}_{0}(n) .
$$

Elle est alors appliquée pour le pas en cours. Toutes ces étapes sont reconduites à chaque pas de temps de régulation.

La prise en compte de contraintes diverses est aisée :

- Les contraintes sur les entrées (par exemple, gradient limite de variation, bande morte de variation) sont intégrées soit à travers la structuration de la loi de commande soit par application directe sur le résultat.

- Les contraintes sur les sorties (par exemple, respect de marnage, gradient limite de niveau en un point quelconque de la retenue) sont gérées par une stratégie de supervision, solution plus simple qu'une intégration directe aux critères.

\subsection{Application}

Cette dernière méthode est en cours de développement et sera installée dans les calculateurs de 12 aménagements du Rhône en aval de Lyon dans le cadre du projet commun EDF/CNR dénommé Rhône 2000 . Ce projet va permettre la rénovation de l'ensemble des automatismes du Bas-Rhône. La régulation par commande prédictive décrite ci-dessus aura pour tâche de réguler les retenues en période de crue en garantissant la sécurité des biens et des personnes le long du fleuve.

Des premiers tests du logiciel de régulation ont été réalisés pour l'aménagement de Péage-de-Roussillon. Les hydrogrammes de la figure 6 montrent l'efficacité de la commande prédictive comparativement à une régulation PID classique.

Dans l'essai présenté ci-contre (fig.6), le plan d'eau doit être baissé. Une contrainte importante est alors la limitation volontairement imposée du gradient de niveau au point de réglage. Avec le PID, le respect de cette contrainte est 


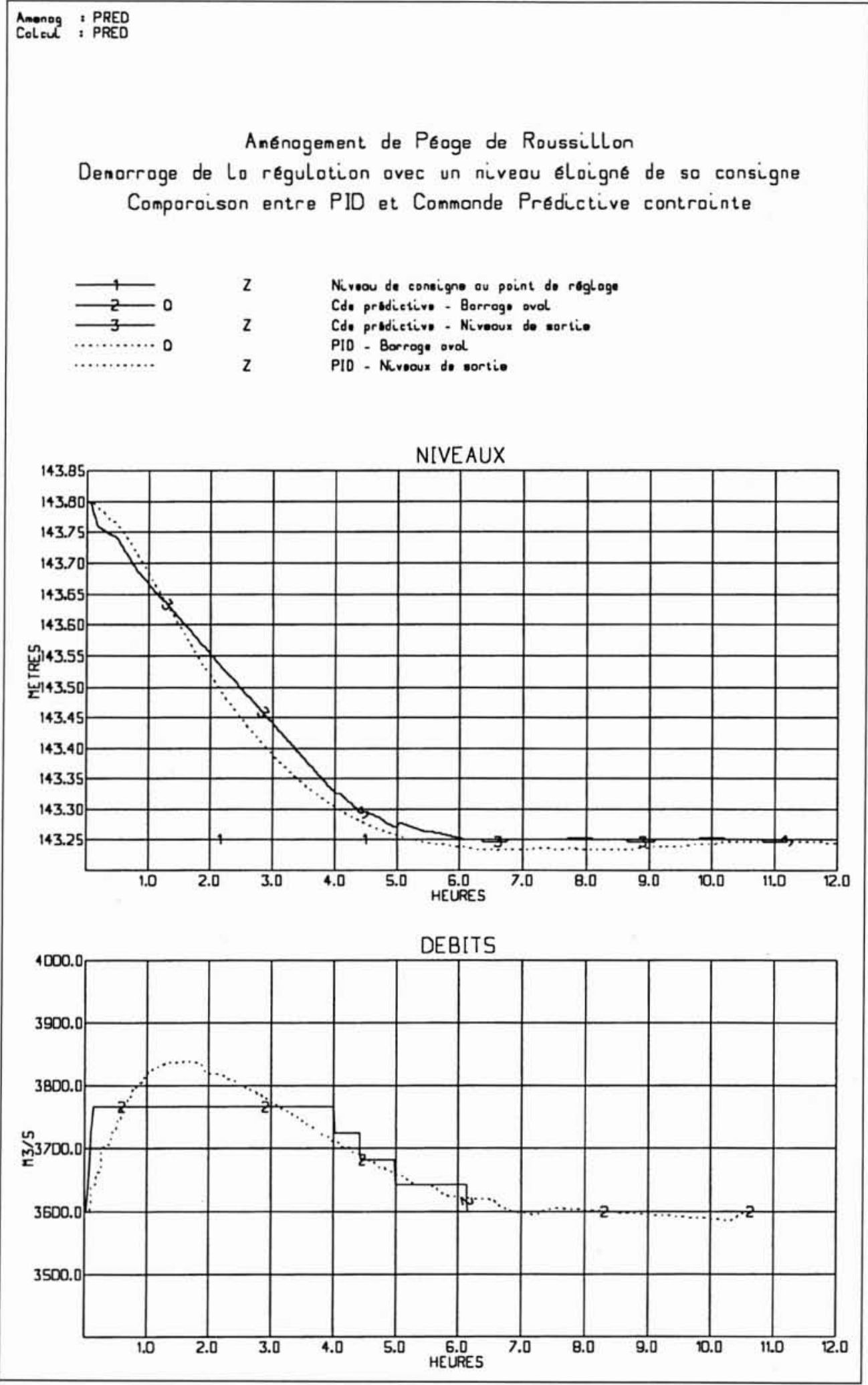

6. Hydrogrammes des débits de commande avec PID et Commande Prédictive.

obtenu par un amollissement du réglage du correcteur mais n'est pas directement contrôlable. La commande prédictive permet quant à elle d'intégrer en amont la limitation du gradient de niveau. La réponse en débit (commande) est caractéristique et très différente de celle du PID, la correction est rapide mais limite les actions sur les organes de commande, ce qui accroît leur durée de vie. De plus, le surdébit est réduit de $30 \%$.

\section{PERSPECTIVES : CONDUITE CEN- TRALISÉE D'UNE CHAÎNE D'AMÉ- NAGEMENTS}

L'optimisation de la gestion des volumes le long des fleuves en période de crue a toujours été un souci des exploitants. Les procédures actuelles permettent déjà un amortissement 
naturel des crues mais restent, pour raison de sécurité primaire, locales à chaque aménagement. Une procédure unique de gestion globale de l'ensemble des aménagements ne pourrait qu'améliorer cet amortissement.

Afin de mettre en œuvre cette méthode, un système permettant de conduire automatiquement une chaîne d'aménagements de manière centralisée en période de crue devra être conçu.

Ces méthodes de régulation multi-variables sont déjà utilisées pour certains canaux d'irrigation où les contraintes sont plus limitées et où les objectifs sont simples. Toute la difficulté de conduire une chaîne de retenues sur un fleuve consiste à hiérarchiser et à intégrer un grand nombre de contraintes et d'objectifs contradictoires.

Pour tester la faisabilité d'une telle approche, nous avons développé un système de conduite par commande prédictive multi-variable et supervision des objectifs et contraintes hiérarchisées (voir Pagès [8]).

Pour piloter plusieurs aménagements, nous avons construit un modèle multi-entrée/multi-sortie par agrégation des modèles d'état des aménagements du Bas-Rhône (voir le chapitre précédent).

L'ensemble de la démarche mathématique d'obtention de la commande vue précédemment est réalisée avec des variables de dimension supérieure, ce qui nous permettra de déterminer le vecteur des débits de commande.

Outre les contraintes spécifiques de chaque aménagement, la gestion globale doit limiter les débits propagés le long du fleuve. Pour ce faire nous avons assigné à chaque retenue un volume utile se traduisant par une zone de marnage pour le niveau de consigne.

Le principal objectif de la conduite centralisée devient ainsi de trouver le meilleur jeu de commandes tel que les niveaux à réguler restent dans leurs zones de marnage et que la pointe du débit propagé soit amortie au mieux le long du cours d'eau. Bien entendu, la présence de perturbations non mesurées et d'affluents capricieux accroît la difficulté.

La sécurité devant être garantie en cas de dégradation (ruptures de liaison ou pannes de la conduite centralisée), nous proposons une structure formée par des régulateurs locaux indépendants mais pilotés par le biais de consignes évolutives à l'intérieur de la zone de marnage citée plus haut. Ces dernières seront élaborées en temps réel par un poste central équipé du système de conduite global par commande prédictive multi-variable puis transmises à chaque régulateur local pour application.

En attendant de pouvoir tester cette méthode sur le Rhône, nous avons construit un chaînage de 6 modèles linéaires identifiés (fig. 8). Pour obtenir les courbes (fig.9), nous avons appliqué une crue $\left(1000 \mathrm{~m}^{3} / \mathrm{s}-\right.$ gradient de montée de $500 \mathrm{~m}^{3} / \mathrm{s} / \mathrm{h}$, de descente de $330 \mathrm{~m}^{3} / \mathrm{s} / \mathrm{h}$ ) sur un affluent se jetant dans la retenue $\mathrm{n}^{\circ} 3$.

Les graphes de la figure 9 représentent la simulation du passage de la crue avec une régulation locale hors gestion centralisée pour le premier et avec la stratégie de conduite centralisée pour le second.

Dans le premier cas, seuls les aménagements situés à l'aval de l'affluent agissent. La crue est reconduite sans amortissement, la retenue ne disposant pas du degré de liberté du marnage (zone de variation du niveau au point de référence).

Pour le second résultat, l'ensemble de la zone de marnage en niveau est utilisée et les aménagements amonts participent à l'action de limitation de la pointe de débit. La réduction du débit maximum obtenue à l'aval est de l'ordre de $45 \%$.

\section{$V \square$ CONCLUSION}

La puissance et la souplesse des moyens informatiques permettent aujourd'hui d'utiliser des modèles numériques très précis.

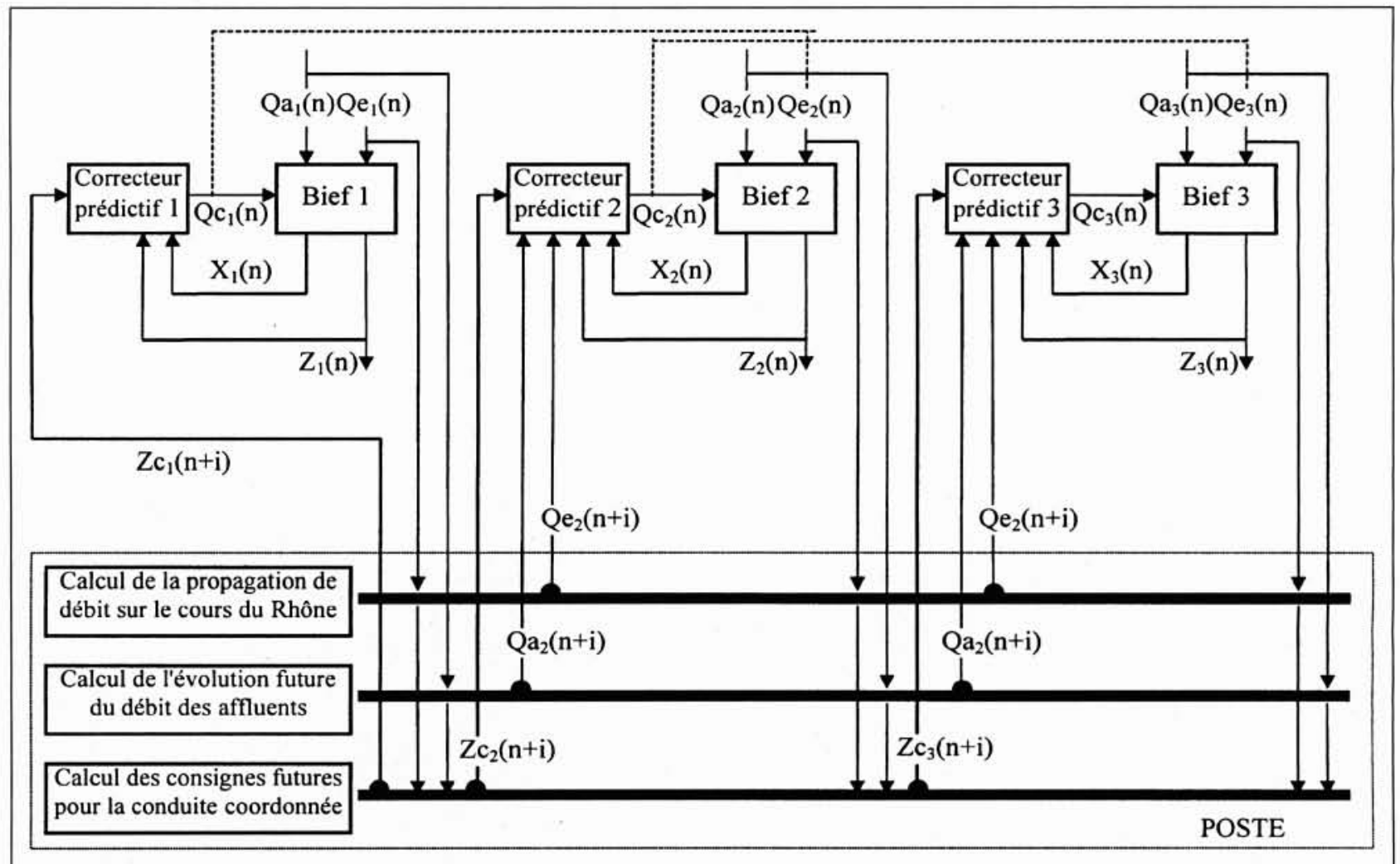

7. Conduite centralisée des régulateurs locaux $-Q e:$ débit entrant $-Q a:$ débit affluent $-Q c:$ débit de commande $Z$ : niveau réel $-Z c$ : niveau de consigne $-X:$ vecteur d'état. 


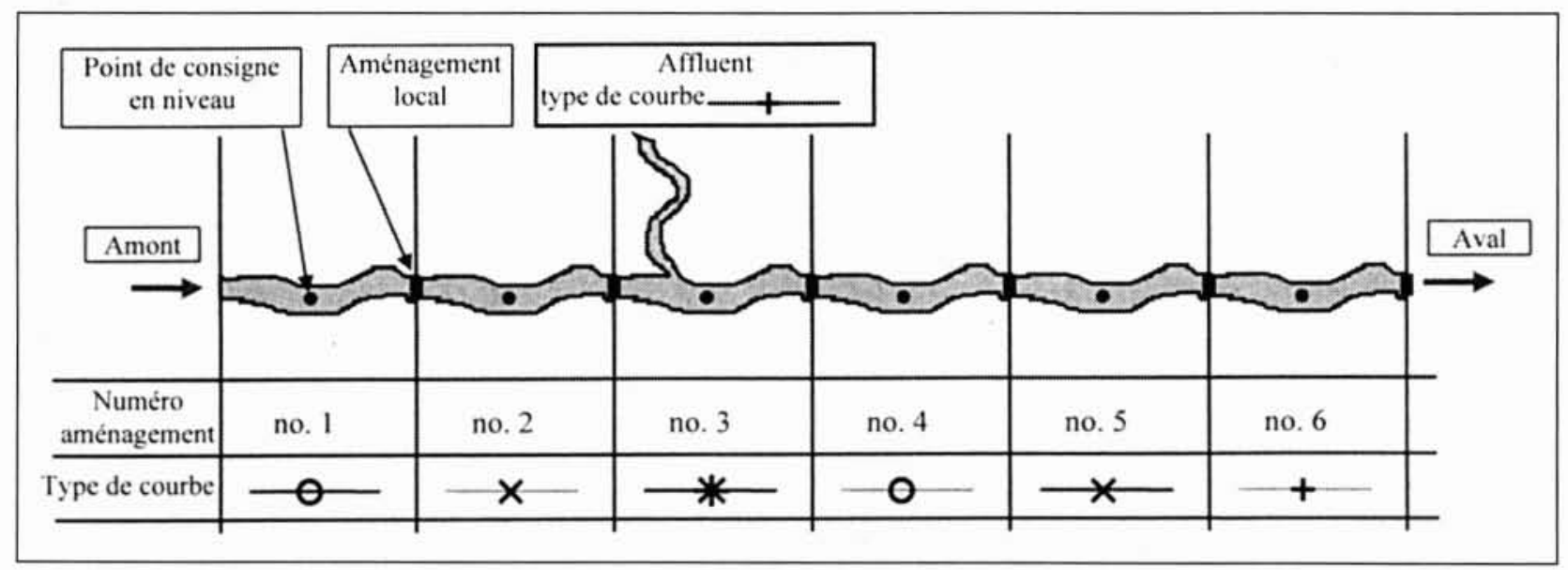

8. Schéma de la portion de fleuve reconstruite pour la simulation.
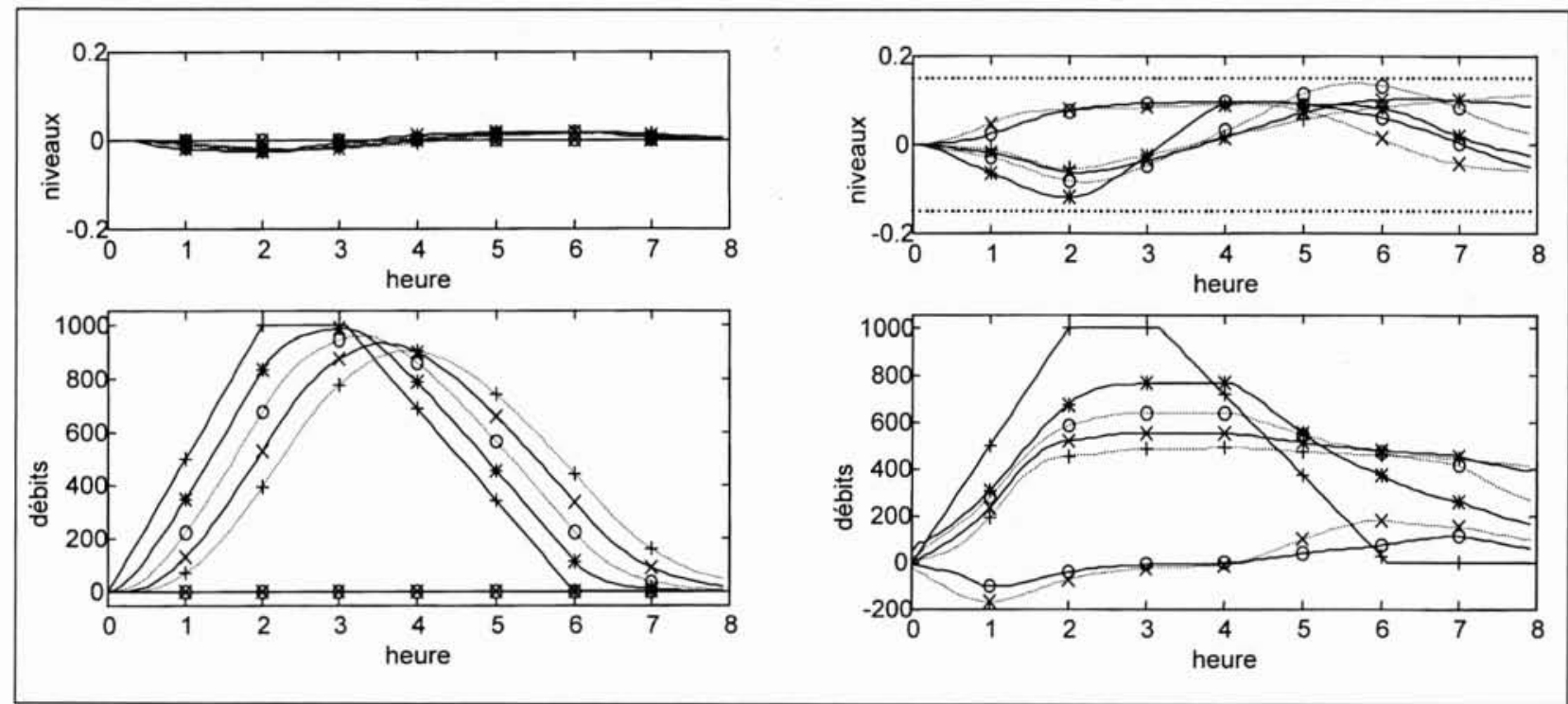

9. Résultats de simulations en régulation locale et en conduite coordonnée.

La CNR a choisi de développer en interne son code de simulation des écoulements à surface libre monodimensionnels.

Grâce à la parfaite maîtrise de ce produit, un logiciel de régulation par commande prédictive avec modèle mathématique embarqué a ensuite été réalisé. La première campagne de test en simulation a révélé un excellent comportement. Son déploiement sur 12 sites accompagné des essais sur plate-forme adaptés est prévu dans les années à venir.

Dans une seconde phase, une régulation globale coordonnée est envisagée. L'étude de faisabilité présentée dans ce document montre son fort potentiel en matière de réduction des pointes de débit en crue. Il reste cependant un important travail pour pouvoir valider complètement cette méthode, en particulier dans les cas de fonctionnements dégradés.

\section{BIBLIOGRAPHIE}

[1] Richalet J. - «Pratique de la commande prédictive», 1993, Hermès.

[2] Cunge J.A., Holly F.M., Verwey A. - «Practical Aspects of computational river hydraulics», 1980, Pitman.
[3] Roult D., Roumieu P. - «Logiciel CRUE: Modèle de connaissance embarqué et boîte à outils pour des études hydrauliques et de régulation», 1997, CNR, RIC 97, Marrakesh.

[4] Pages J.C. - «Introduction à la régulation du Rhône par commande prédictive avec modèle mathématique embarqué», 1995, CNR, DEA de l'Institut des Sciences et Techniques de I'Ingénieur de Lyon, UCBL I.

[5] LANDAU I.D. - «Identification et commande des systèmes linéaires», 1988, Hermès.

[6] LAGoutte J.R. - «La régulation en chaine des aménagements du Rhône Français de la frontière Suisse à la mer». 1995, CNR, Wasser Energie Luft, 87.Jahrgang, Heft 3/4. CH-5401 Baden.

[7] COMpas J.M., PAgeS J.C. - «Régulation par commande prédictive et modèle de connaissance embarqué», 1997. CNR, International Workshop on Regulation of Irrigation Canals 97, Marrakesh.

[8] Pages J.C., Compas J.M., SAu J. - «Conduite coordonnée d'une chaîne d'aménagements hydrauliques à base de commande prédictive», 1997, CNR et ISTIL, RIC 97, Marrakesh. 